\title{
Editorial
}

\section{Innovations in Communications Security}

\author{
Mazdak Zamani, ${ }^{1}$ Isao Echizen, ${ }^{2}$ and Arash Habibi Lashkari ${ }^{3}$ \\ ${ }^{1}$ Kean University, Union, NJ, USA \\ ${ }^{2}$ National Institute of Informatics, Tokyo, Japan \\ ${ }^{3}$ University of New Brunswick, Fredericton, NB, Canada \\ Correspondence should be addressed to Mazdak Zamani; zamani.mazdak@gmail.com
}

Received 12 July 2016; Accepted 12 July 2016

Copyright (C) 2016 Mazdak Zamani et al. This is an open access article distributed under the Creative Commons Attribution License, which permits unrestricted use, distribution, and reproduction in any medium, provided the original work is properly cited.

Communications security is the discipline of preventing unauthorized interceptors from accessing telecommunications in an intelligible form, while still delivering content to the intended recipients.

This special issue collects 6 papers from 15 authors belonging to different countries and institutions. It summarizes the most recent developments and ideas on emerging communications security, with particular focus on privacy preserving, key distribution scheme, and digital image watermarking.

In the paper by $\mathrm{X}$. Cao et al. entitled "A PrivacyPreserving Outsourcing Data Storage Scheme with Fragile Digital Watermarking-Based Data Auditing," a privacypreserving and auditing-supporting outsourcing data storage scheme by using encryption and digital watermarking is proposed which combines digital watermark technology with encryption method for outsourcing data storage.

In the paper by $\mathrm{H}$. Zhong et al. entitled "An Efficient Electronic English Auction System with a Secure On-Shelf Mechanism and Privacy Preserving," a novel electronic English auction system is proposed which uses symmetrical encryptions and fewer ECC operations and improves the security and reduces the system cost.

In the paper by J. N. Luo and M. H. Yang entitled "Analysis and Improvement of Key Distribution Scheme for Secure Group Communication," a scheme to enhance the security of EGK is proposed which guarantees forward and backward secrecy, prevents message modification and forgery during rekeying, requires sender verification, and therefore prevents MITM attacks in group communication.

In the paper by Y. Zolotavkin and M. Juhola entitled "A New Scalar Quantization Method for Digital Image Watermarking," a new scalar QIM-based watermarking method is proposed which provides higher robustness under AWGN and GA compared to other quantization methods. The advantage of the method is due to the introduced procedure of recovery after GA as well as new distribution of quantized samples with IDL.

In the paper by M. C. Tran and Y. Nakamura entitled "Communication Behaviour-Based Big Data Application to Classify and Detect HTTP Automated Software," a new method is proposed to detect and classify auto-ware communication behaviour based on HTTP traffic that uses minor features in HTTP traffic and does not use any signature or content-based feature.

In the paper by J. Wang and J. Liu entitled "The Comparison of Distributed P2P Trust Models Based on Quantitative Parameters in the File Downloading Scenarios," a new method is proposed to compare and evaluate the trust models with quantitative parameters in P2P file downloading scene that evaluated parameters extracted from the trust related concepts and modelled into a hierarchical structure.

Mazdak Zamani Isao Echizen Arash Habibi Lashkari 


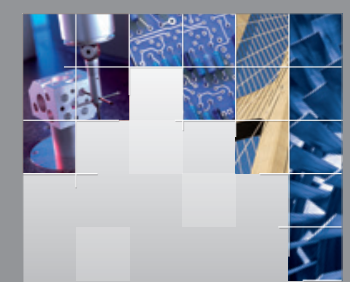

\section{Enfincering}
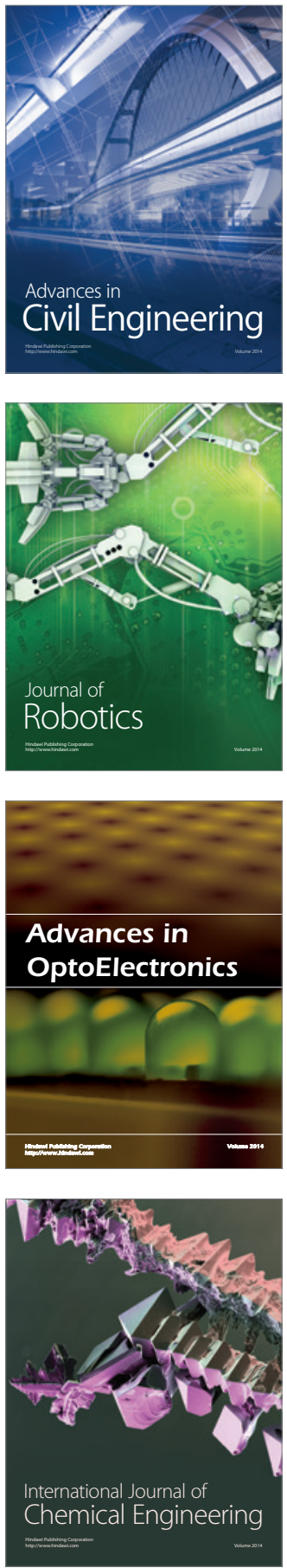

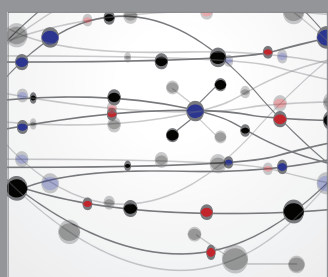

The Scientific World Journal

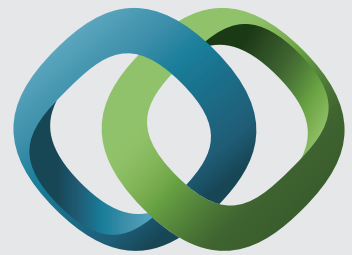

\section{Hindawi}

Submit your manuscripts at

http://www.hindawi.com
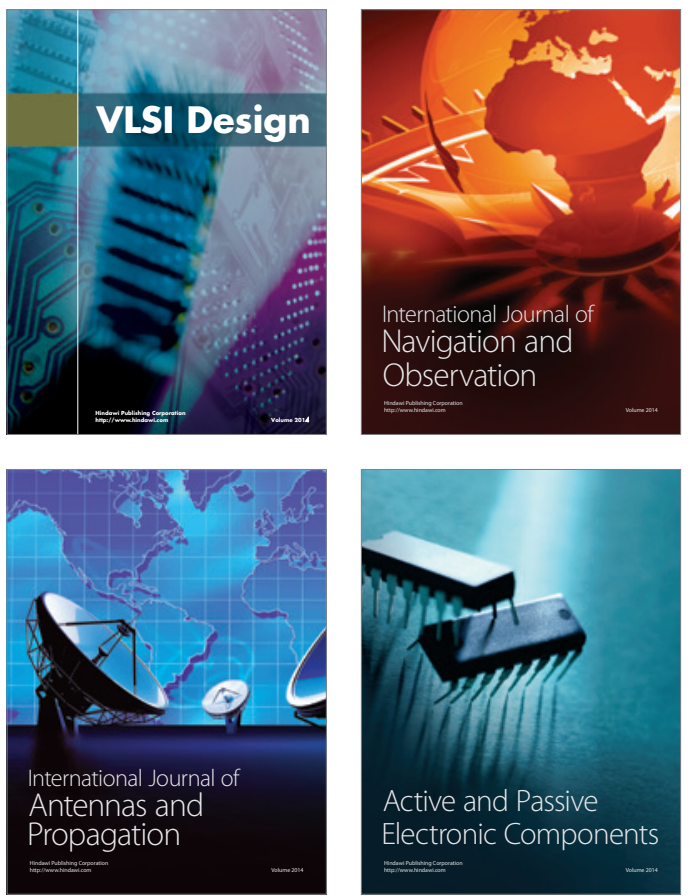
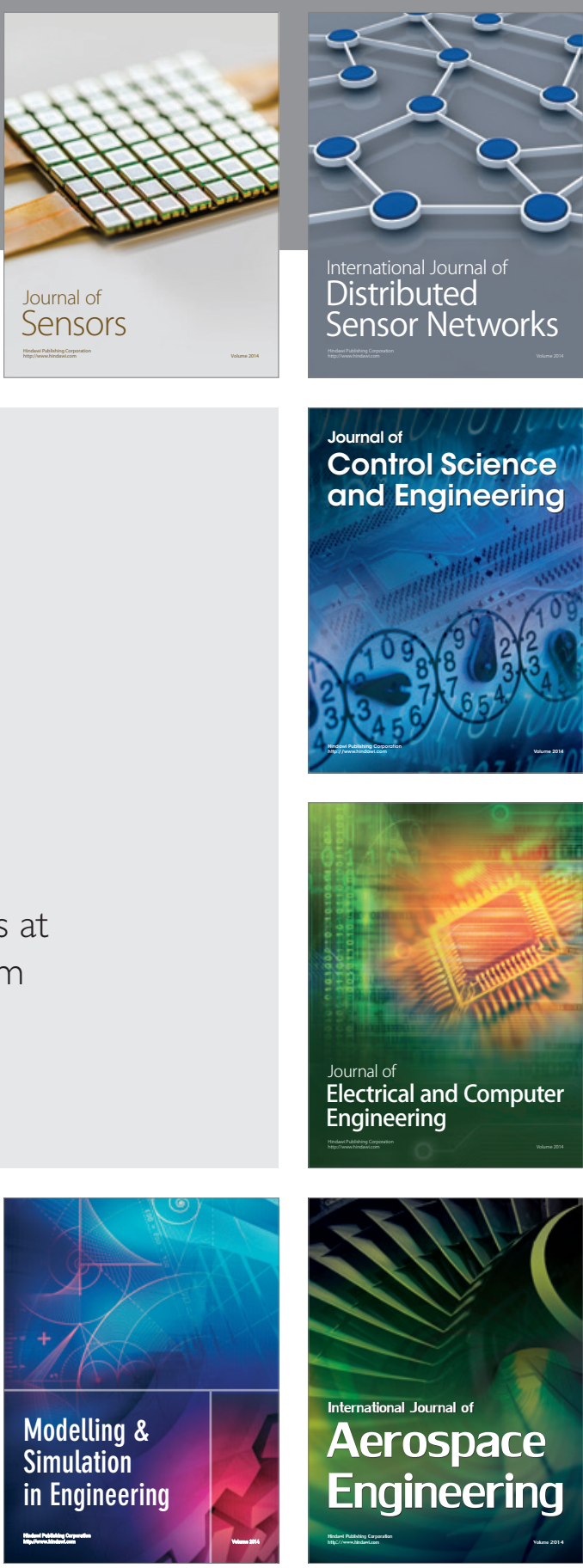

International Journal of

Distributed

Sensor Networks

Journal of

Control Science

and Engineering
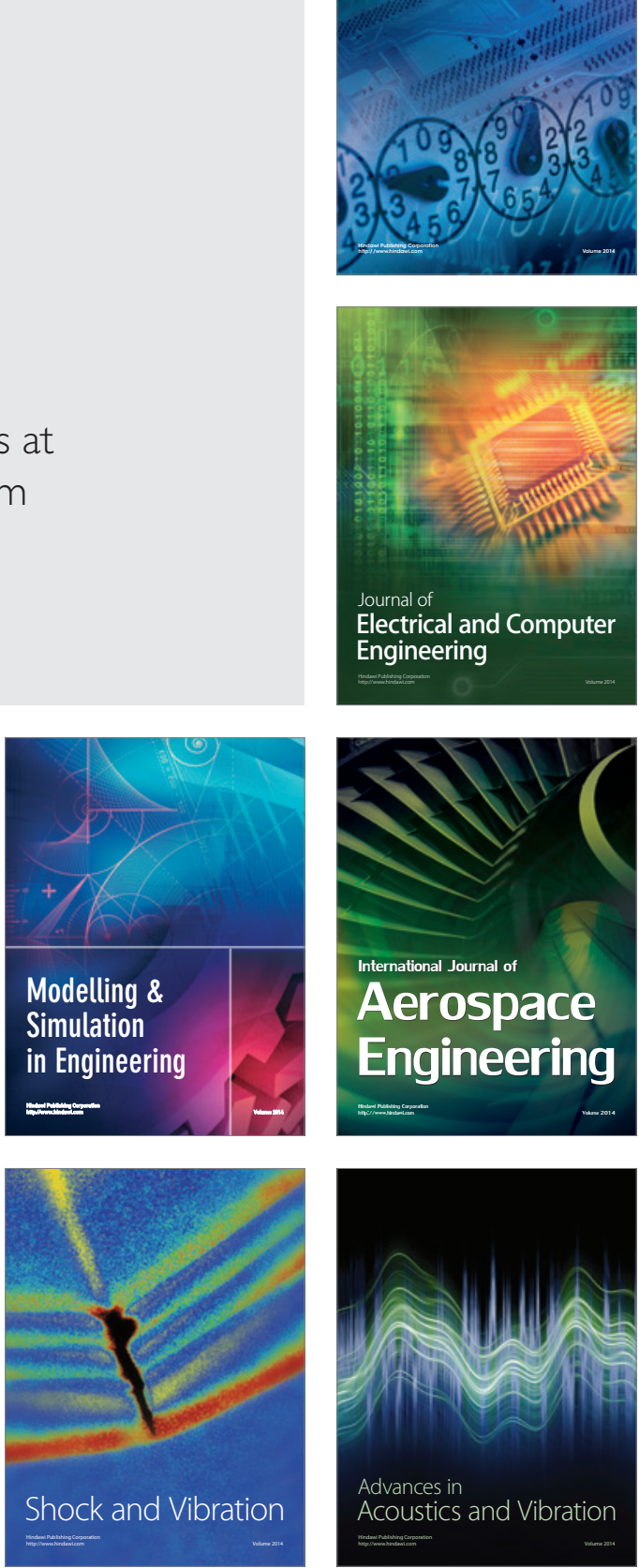